\title{
Video Article \\ Employing Pressurized Hot Water Extraction (PHWE) to Explore Natural Products Chemistry in the Undergraduate Laboratory
}

\author{
Curtis C. Ho ${ }^{1}$, Bianca J. Deans* ${ }^{*}$, Jeremy Just* ${ }^{*}$, Gregory G. Warr ${ }^{2}$, Shane Wilkinson ${ }^{2}$, Jason A. Smith ${ }^{1}$, Alex C. Bissember ${ }^{1}$ \\ ${ }^{1}$ School of Natural Sciences - Chemistry, University of Tasmania \\ ${ }^{2}$ School of Chemistry, The University of Sydney \\ * These authors contributed equally
}

Correspondence to: Shane Wilkinson at shane.wilkinson@sydney.edu.au, Jason A. Smith at jason.smith@utas.edu.au, Alex C. Bissember at alex.bissember@utas.edu.au

URL: https://www.jove.com/video/58195

DOI: doi:10.3791/58195

Keywords: Chemistry, Issue 141, Chemical Education, Pressurized Hot Water Extraction, Natural Products, Undergraduate Laboratory, Bioprospecting, Organic Chemistry

Date Published: 11/7/2018

Citation: Ho, C.C., Deans, B.J., Just, J., Warr, G.G., Wilkinson, S., Smith, J.A., Bissember, A.C. Employing Pressurized Hot Water Extraction (PHWE) to Explore Natural Products Chemistry in the Undergraduate Laboratory. J. Vis. Exp. (141), e58195, doi:10.3791/58195 (2018).

\section{Abstract}

A recently developed pressurized hot water extraction (PHWE) method which utilizes an unmodified household espresso machine to facilitate natural products research has also found applications as an effective teaching tool. Specifically, this technique has been used to introduce second- and third-year undergraduates to aspects of natural products chemistry in the laboratory. In this report, two experiments are presented: the PHWE of eugenol and acetyleugenol from cloves and the PHWE of seselin and (+)-epoxysuberosin from the endemic Australian plant species Correa reflexa. By employing PHWE in these experiments, the crude clove extract, enriched in eugenol and acetyleugenol, was obtained in $4-9 \% \mathrm{w} / \mathrm{w}$ from cloves by second-year undergraduates and seselin and (+)-epoxysuberosin were isolated in yields of up to $1.1 \% \mathrm{w} / \mathrm{w}$ and $0.9 \%$ $\mathrm{w} / \mathrm{w}$ from $\mathrm{C}$. reflexa by third-year students. The former exercise was developed as a replacement for the traditional steam distillation experiment providing an introduction to extraction and separation techniques, while the latter activity featured guided-inquiry teaching methods in an effort to simulate natural products bioprospecting. This primarily derives from the rapid nature of this PHWE technique relative to traditional extraction methods that are often incompatible with the time constraints associated with undergraduate laboratory experiments. This rapid and practical PHWE method can be used to efficiently isolate various classes of organic molecules from a range of plant species. The complementary nature of this technique relative to more traditional methods has also been demonstrated previously.

\section{Video Link}

The video component of this article can be found at https://www.jove.com/video/58195/

\section{Introduction}

The isolation and identification of natural products are of fundamental importance to the scientific community and society more generally. ${ }^{1}$ Bioprospecting, the search for valuable organic molecules found in nature,remains an indispensable process in the discovery of new drug leads and potential therapeutic agents. It is estimated that, from $1981-2014, \sim 75 \%$ of all approved small molecule pharmaceutical drugs were natural products, natural product-derived or natural product-inspired. ${ }^{1}$ Furthermore, natural products possess enormous structural and chemical diversity. For this reason, they also represent valuable chemical scaffolds that can be directly used in organic synthesis or in the development of chiral ligands and catalysts. ${ }^{2,3}$

Traditionally, relatively time-intensive procedures such as maceration, Soxhlet extraction, and steam distillation have been the mainstay of research focused on the isolation of secondary metabolites from plants. ${ }^{4}$ More modern extraction techniques, including accelerated solvent extraction, have focused on reducing extraction times and establishing greener protocols. ${ }^{4,5}$ In 2015 , an original pressurized hot water extraction (PHWE) method was reported. ${ }^{6}$ This technique employed an unmodified household espresso machine to facilitate the rapid and particularly efficient extraction of shikimic acid from star anise. Espresso machines have been specifically designed and engineered to extract organic molecules from appropriately ground coffee beans. To achieve this, these instruments heat water at temperatures up to $96^{\circ} \mathrm{C}$ and at pressures of typically 9 bar. $^{7}$ With this in mind, it is perhaps not surprising that espresso machines can be utilized to efficiently extract natural products from a range of plant material.

Subsequent studies involving a variety of terrestrial plant species have demonstrated the capacity of this PHWE technique to efficiently extract natural products across a relatively broad polarity range. ${ }^{6,8,9,10,11,12,13,14,15}$ Furthermore, compounds containing somewhat sensitive functional groups, such as aldehydes, epoxides, glycosides, and potentially epimerizable stereogenic centers were typically unaffected by the extraction process. The complementary nature of this technique relative to more traditional methods has also been demonstrated. ${ }^{12,16}$ This PHWE method has also been employed to isolate multi-gram quantities of natural products, which have been used to prepare novel natural product derivatives and in complex molecule synthesis more generally., 81,17 
It was identified that this new PHWE method could serve as a useful teaching tool that could be incorporated in the undergraduate laboratory. This primarily derives from the rapid nature of this technique relative to the traditional extraction methods that are often incompatible with the time constraints associated with undergraduate laboratory experiments. Consequently, this technique supplanted the traditional undergraduate chemistry laboratory experiment focused on the extraction of eugenol from cloves employing steam-distillation at the University of Tasmania. 9,18 Since that time, variations of this experiment have been adopted by other universities and a modified experiment focusing on the PHWE of cloves now features in the undergraduate chemistry laboratory program at the University of Sydney (vide infra).

In order to demonstrate the practicality and feasibility of employing this new PHWE approach for teaching purposes, two protocols are presented as part of this study. The first part of this report highlights an experiment on the PHWE of eugenol and acetyleugenol from cloves which is part of the second-year undergraduate laboratory program at the University of Sydney (Figure 1). This experiment serves to introduce students to natural products chemistry while developing fundamental practical skills. The second part features an experiment on the PHWE of the endemic Australian plant species Correa reflexa which is part of the third-year undergraduate laboratory program at the University of Tasmania (Figure 2) This experiment is designed to simulate natural products bioprospecting and reinforce core laboratory techniques. ${ }^{11}$

\section{Protocol}

NOTE: It is advisable that all procedures are performed in a fume hood. Students must wear appropriate personal protective equipment at all times in the laboratory and the safety data sheets (SDS) associated with each reagent must be consulted before use.

\section{PHWE of cloves: isolation of eugenol and acetyleugenol}

1. Extraction of eugenol and acetyleugenol from cloves

1. Place coarsely ground cloves $(12.5 \mathrm{~g})$ in a $250-\mathrm{mL}$ beaker.

2. Add sand $(12.5 \mathrm{~g})$ to the clove grinds and mix well.

3. Collect a portafilter (sample compartment) and load the basket with the entire clove-sand mixture. Lightly compress the sample with the tamper.

NOTE: Do not compress the mixture too much or fluid will not flow through.

4. Position the portafilter into the espresso machine and place a clean $250-\mathrm{mL}$ beaker beneath it. Add a $30 \%$ ethanol/ $\mathrm{H}_{2} \mathrm{O}$ solution to the water tank of the espresso machine if it is less than half full.

5. Use the espresso machine to collect $100 \mathrm{~mL}$ of the extract.

NOTE: Consult an instructor if the machine appears to be clogged.

6. Allow the portafilter to finish dripping and then remove it from the espresso machine. CAUTION: The grinds and surrounding metal areas will be hot.

7. Using a spatula, remove the clove grinds from the portafilter and discard into the waste bin.

8. Rinse out the residual solids from the portafilter with $\mathrm{H}_{2} \mathrm{O}$ under a tap in the sink and return it for the next person to use.

9. Cool the clove extract in an ice bath until the temperature has reduced to at least $30^{\circ} \mathrm{C}$.

10. Place the extract into a $250-\mathrm{mL}$ separatory funnel, add $30 \mathrm{~mL}$ of hexane and shake gently.

11. Place the separating funnel in a ring clamp fitted to a retort stand and allow the aqueous and organic layers to separate then collect the aqueous (lower) layer back into the $250-\mathrm{mL}$ beaker.

NOTE: It can take to 10 minutes for the layers to separate. Students are advised to carry out solvent optimization of TLCs while waiting for the first separation to occur (see steps 1.2).

12. Transfer the organic (top) layer (which contains the product) to a clean $250-\mathrm{mL}$ conical flask, and then pour the bottom (aqueous) layer back into the separatory funnel.

13. Extract the aqueous layer a further two times with hexane $(2 \times 30 \mathrm{~mL})$.

14. Combine the organic (top) layers into the same flask after each extraction.

15. After the third liquid-liquid extraction, pour the combined organic extract into the separatory funnel, and wash with $100 \mathrm{~mL}$ of $\mathrm{H}_{2} \mathrm{O}$ by shaking vigorously. Collect the organic (top) layer into a clean $250-\mathrm{mL}$ conical flask and dry by adding $\mathrm{MgSO}_{4}$ and swirling the flask.

16. Filter the ensuing mixture through fluted filter paper contained in a glass funnel into a pre-weighed $250-\mathrm{mL}$ round-bottom flask. NOTE: The solid residue (hydrated $\mathrm{MgSO}_{4}$ ) can be discarded in the waste.

17. Evaporate the solvent (hexane) from the collected filtrate using a rotary evaporator (water bath temperature: $60^{\circ} \mathrm{C}$, vacuum pressure: $350 \mathrm{mbar}$ ) and re-weigh the flask containing the resultant oil.

2. Optimization of thin-layer chromatography (TLC) solvent system

NOTE: As a group, students will be assigned a solvent system from 100:0 acetone:cyclohexane to 0:100 acetone:cyclohexane by the instructor to identify the ratio that provides the maximum resolution of eugenol from acetyleugenol.

1. Obtain a TLC reference solution of pure eugenol and acetyleugenol. NOTE: The necessary TLC reference solutions of pure eugenol and acetyleugenol were prepared by lab technicians prior to the lab session.

2. On a TLC plate, mark a baseline $\sim 1.5 \mathrm{~cm}$ from the bottom with a soft pencil. Mark off three equally spaced points.

3. Use a TLC spotter to spot one drop of pure eugenol TLC reference solution in one lane, one spot of pure acetyleugenol TLC reference solution in the third lane and a spot of each in the second lane (the co-spot).

4. Check for the presence of eugenol and acetyleugenol on the TLC plate by viewing the plate under a UV lamp (254 $\mathrm{nm}$ ) in the TLC viewing cabinet.

NOTE: There should be small (1-2 mm wide) black spots on the plate where the TLC reference solutions were spotted. If there are no spots or the spots appear faint, apply another spot of the appropriate TLC solution until a black spot is observed under UV light.

5. Add $10 \mathrm{~mL}$ of the allocated solvent mixture to a clean, dry TLC jar. NOTE: Ensure the solvent height in the jar does not exceed $\sim 1 \mathrm{~cm}$

6. Using tweezers, place the prepared TLC plate into the TLC jar. Close the lid of the jar. NOTE: The solvent must lie below the baseline of the TLC plate. 
7. Allow the solvent to travel up the TLC plate. Once the solvent is $\sim 1 \mathrm{~cm}$ from the top of the plate, remove the TLC plate from the jar with tweezers and mark the line of the solvent front with a pencil.

8. Allow the solvent to evaporate from the TLC plate $(\sim 1 \mathrm{~min})$ then view the TLC plate under a UV lamp $(254 \mathrm{~nm})$. Using a pencil, circle the black spots observed on the TLC plate.

9. Calculate the retention factor $\left(\mathrm{R}_{f}\right)$ of eugenol and acetyleugenol by dividing the distance travelled by the compound by the distance travelled by the solvent.

10. Calculate the difference between the $R_{f}$ values for eugenol and acetyleugenol $\left(\Delta R_{f}\right)$.

11. Share the results with the rest of the class. Record the retention values acquired by other students with other solvent ratios.

12. Identify which solvent system will be best to analyze the crude eugenol solution and subsequent purification steps.

NOTE: The best TLC solvent ratio will provide the greatest separation between eugenol and acetyleugenol denoted by the largest $\Delta \mathrm{R}_{f}$ value. If $\Delta R_{f}$ is plotted against solvent composition, the graph should resemble a bell curve.

3. Separation of eugenol and acetyleugenol by liquid-liquid extraction

1. Add hexane $(10 \mathrm{~mL})$ to the crude eugenol-containing extract obtained from step 1.1.17, and pour the ensuing solution into a $250-\mathrm{mL}$ separatory funnel.

2. Rinse the round-bottom flask with hexane $(10 \mathrm{~mL})$ and add this to the separatory funnel.

3. Extract the hexane solution with $3 \mathrm{M}$ aqueous $\mathrm{NaOH}(2 \times 25 \mathrm{~mL})$ via liquid-liquid extraction. Collect and combine the aqueous bottom layers in a $250-\mathrm{mL}$ conical flask from each extraction. Collect the organic layer in a $50-\mathrm{mL}$ conical flask and dry by adding $\mathrm{MgSO}_{4}$ and swirling the flask.

CAUTION: $\mathrm{NaOH}$ is corrosive. Avoid any contact with skin.

NOTE: Acetyleugenol remains in the organic layer, while eugenol is now in the alkaline aqueous extract (bottom layers).

4. Retain the organic layer (organic solution A) for later TLC analysis.

5. Swirl the conical flask that contains the alkaline aqueous fraction from step 1.3.3 in an ice-water bath and slowly add $10 \mathrm{M}$ aqueous $\mathrm{HCl}$ until a white emulsion is formed; check its acidity with Congo red paper, using a pipette to transfer a drop of the solution onto the $\mathrm{pH}$ paper (it should turn blue).

CAUTION: $\mathrm{HCl}$ is corrosive. Avoid any contact with skin. Addition of $\mathrm{HCl}$ can cause vigorous bubbling, $\mathrm{HCl}$ should be added carefully, keeping the conical flask on ice.

NOTE: A total of $20-30 \mathrm{~mL}$ of $\mathrm{HCl}(10 \mathrm{M}$ of an aqueous solution) will be required.

6. Extract the milky aqueous emulsion with hexane $(2 \times 30 \mathrm{~mL})$, using liquid-liquid extraction in a $250 \mathrm{~mL}$ separating flask. Make sure that the temperature of the aqueous extract is at room temperature or below before adding the hexane. Combine the two hexane extracts into a clean $100 \mathrm{~mL}$ conical flask. NOTE: The eugenol will now be in the combined organic (top) layers (organic solution $B$ ).

7. Add $\mathrm{MgSO}_{4}$ to dry organic solution $\mathrm{B}$.

8. Analyze organic solution A, organic solution B, pure eugenol TLC reference and acetyleugenol TLC reference by TLC using the optimized TLC solvent ratio identified in the previous session.

9. Filter organic solution $A$ and $B$ through fluted filter paper into separate pre-weighed $250-\mathrm{mL}$ round-bottom flasks. Discard the solid residue (hydrated $\mathrm{MgSO}_{4}$ ) in the waste.

10. Remove the solvent from the round-bottom flasks using a rotary evaporator (water bath temperature: $60{ }^{\circ} \mathrm{C}$, vacuum pressure: 350 mbar).

11. Add diethyl ether $(5 \mathrm{~mL})$ to each round-bottom flask and transfer the purified acetyleugenol (organic solution A) and eugenol (organic solution $\mathrm{B}$ ) into an unlabeled, preweighed vial using a funnel.

12. Rinse the flask with further diethyl ether $(5 \mathrm{~mL})$ into the vial. Evaporate the solvent using a rotary evaporator (water bath temperature: $50^{\circ} \mathrm{C}$, vacuum pressure $800 \mathrm{mbar}$ ) with a vial attachment. Record the yield and label the vial appropriately.

13. Analyze organic solution A, organic solution B, pure eugenol TLC reference and acetyleugenol TLC reference by TLC using the optimized TLC solvent ratio identified in the previous session.

NOTE: Aqueous solutions can be poured down the sink for disposal. Hexane and ether waste should be disposed of in the nonchlorinated organic waste bottles.

\section{PHWE of Correa reflexa : isolation of seselin and (+)-epoxysuberosin}

\section{Session 1. PHWE of Correa reflexa}

1. Grind Correa reflexa leaves $(10 \mathrm{~g})$ in an electric spice grinder and then transfer the ground plant material to a $250-\mathrm{mL}$ beaker. NOTE: Grinding should take $20-30$ seconds.

2. Add $\sim 2 \mathrm{~g}$ of coarse sand to the beaker containing plant material.

3. Mix and pack into the basket of the portafilter (sample compartment). Compress the sample with the tamper. NOTE: Do not pack the sample too tightly.

4. Add $\sim 300 \mathrm{~mL}$ of $35 \%$ ethanol/ $/ \mathrm{H}_{2} \mathrm{O}$ solution to the espresso machine tank.

5. Position the portafilter into the espresso machine and place a clean $250-\mathrm{mL}$ beaker beneath it.

6. Collect $\sim 100 \mathrm{~mL}$ of extract, wait for $\sim 1$ minute and then collect a further $100 \mathrm{~mL}$. CAUTION: The machine and extracts will be hot at this point.

7. Cool this mixture in ice bath and evaporate the ethanol using a rotary evaporator (water bath temperature: $\sim 40{ }^{\circ} \mathrm{C}$ ).

8. Transfer the aqueous extract to a separatory funnel and extract with ethyl acetate $(4 \times 50 \mathrm{~mL})$. NOTE: Time may be needed to allow emulsions to separate between extractions.

9. Combine the organic extracts, dry by adding $\mathrm{MgSO}_{4}$ and swirling the flask, filter using a sintered glass funnel, and evaporate using a rotary evaporator (water bath temperature: $\sim 35^{\circ} \mathrm{C}$ ) to provide the crude extract.

10. Obtain an ${ }^{1} \mathrm{H}$ nuclear magnetic resonance (NMR) spectrum (see the instructor for assistance). ${ }^{11}$

11. Perform TLC analysis of the crude extract to determine an appropriate solvent system to isolate the compounds that have been extracted. 
NOTE: TLC analysis is performed by analogy to procedures outlined in step 1.2 .

2. Session 2. Separation of seselin and (+)-epoxysuberosin by flash column chromatography ${ }^{11,19}$

NOTE: The following protocol involves the use of flash column chromatography for the separation of organic compounds. Please consult an instructor to demonstrate how to pack a flash silica gel column.

1. Place the column ( $\sim 30 \mathrm{~mm}$ in diameter) in a clamp fitted to a retort stand. Place a $100-\mathrm{mL}$ conical flask underneath the column.

2. Fill the column with silica gel $(60 \mu \mathrm{m}$ flash grade) to a level of $\sim 10 \mathrm{~cm}$ and then add hexanes $(\sim 100 \mathrm{~mL})$ to the column.

3. Place a glass stopper in the column, remove the column from the clamp and shake to obtain a slurry. Place the column in the clamp and then allow the mixture to settle.

4. Open the tap of the column and, using a gas adaptor attached to a compressed air line, empty the column to leave $\sim 2 \mathrm{~mm}$ of solvent above the bed of silica gel. Remove the gas adaptor then close the tap.

5. Use the hexanes collected in the conical flask $(\sim 5 \mathrm{~mL})$ to wash down any silica gel from the walls of the column with a Pasteur pipette fitted with a rubber septum.

6. Repeat step 2.2.4 then add a small layer of sand $(\sim 1 \mathrm{~cm})$ to the column.

7. Add dichloromethane $(\sim 1 \mathrm{~mL})$ to the flask containing the crude extract from step 2.1.9. Carefully load the ensuing solution onto the column using a Pasteur pipette fitted with a rubber septum. Open the tap of the column and allow the sample to adsorb onto the silica gel.

8. Repeat step 2.2 .7 a further two times.

9. Carefully add hexanes $(\sim 20 \mathrm{~mL})$ to the column. Repeat step 2.2.4.

10. Carefully add $(\sim 180 \mathrm{~mL}$ of a $15 \%$ ethyl acetate/hexanes solution). Open the tap of the column and, using a gas adaptor attached to a compressed air line, empty the column to leave $\sim 2 \mathrm{~mm}$ of solvent above the bed of silica gel, collecting the fractions into $10-\mathrm{mL}$ test tubes.

NOTE: This will allow seselin to be isolated.

11. Combine the test tube fractions containing seselin in a $250-\mathrm{mL}$ round bottom flask and evaporate using a rotary evaporator (water bath temperature: $\sim 35^{\circ} \mathrm{C}$ ).

NOTE: TLC analysis is used to determine this and is performed by analogy to procedures outlined in step 1.2.

12. Carefully add ( $75 \mathrm{~mL}$ of a $25 \%$ ethyl acetate/hexanes solution). Open the tap of the column and, using a gas adaptor attached to a compressed air line, empty the column to leave $\sim 2 \mathrm{~mm}$ of solvent above the bed of silica gel, collecting the fractions into $10-\mathrm{mL}$ test tubes.

NOTE: This will allow (+)-epoxysuberosin to be isolated.

13. Combine the test tube fractions containing $(+)$-epoxysuberosin in a $250-\mathrm{mL}$ round bottom flask and evaporate using a rotary evaporator (water bath temperature: $\sim 35^{\circ} \mathrm{C}$ ).

NOTE: TLC analysis is used to determine this and is performed by analogy to procedures outlined in step 1.2.

14. Samples of the isolated compounds are analyzed using NMR spectroscopy. ${ }^{11}$ NOTE: NMR spectroscopy experiments are performed by a lab technician.

\section{Representative Results}

PHWE of cloves. When attempting to perform the liquid-liquid extraction step, students often encountered emulsions (the addition of brine was typically not effective). At this stage, students were instructed to allow the mixture to stand in the separating funnel while they explored the effects of eluent composition on the separation of eugenol and acetyleugenol by TLC. It should be noted that hexane can be substituted with either heptane or dichloromethane in the liquid-liquid extraction step. ${ }^{9}$ Students were allocated a TLC solvent ratio of acetone and cyclohexane and provided with pure standards of eugenol and acetyleugenol and then performed TLC analysis (Figure 3). Their results were tabulated on a whiteboard, and the effects of solvent composition on the retention factor $\left(R_{f}\right)$ and the optimum eluent were considered in a group discussion (Table 1). The optimum solvent compositions identified by students typically ranged from $5-20 \%$ acetone/cyclohexane with a $\Delta R_{f}$ between $0.1-0.2$.

Following TLC eluent optimization, students returned to their eugenol extractions. The crude clove extract (consisting mainly of eugenol and acetyleugenol) was isolated in 4-9\% w/w. In the second session of this experiment, students exploited the different acid-base properties of the two major organic molecules to separate them by liquid-liquid extraction. Typically, eugenol was isolated in a yield of $45-65 \% \mathrm{w} / \mathrm{w}$ of the crude extract while acetyleugenol was isolated in a yield of $5-10 \% \mathrm{w} / \mathrm{w}$ of the crude extract. Students then utilized the optimized eluent (identified as outlined above) to determine the success of their liquid-liquid extraction by comparison of their extracts to the pure reference samples by TLC (Figure 3). Students also analyzed their crude clove extract, and their purified eugenol and acetyleugenol samples by performing Fouriertransform infrared (FTIR) spectroscopy. ${ }^{9}$ Solvent or water peaks were occasionally observed in IR spectra due to poorly executed work-up procedures (or poor sample preparation).

Advanced students committed approximately half of their isolated crude oil to the liquid-liquid extraction described above and subjected the other portion to flash column chromatography (more information is provided in the supporting information). Although completing the liquid-liquid extraction and flash column chromatography steps in a single four-hour session may appear rather ambitious this was achievable for most of the advanced students undertaking this experiment. The complete separation of eugenol from acetyleugenol by flash column chromatography was rarely achieved due to their close retention factors (Figure 4). However, students were generally able to collect a few fractions containing pure eugenol. Advanced students were then asked to comment on the two different purification techniques as part of their report. 
PHWE of Correa reflexa. Students performed the PHWE of Correa reflexa with minimal assistance of the laboratory instructor. During the liquidliquid extraction step emulsions typically formed and students often were required to allow the mixture to stand in the separatory funnel $(\sim 0.25 \mathrm{~h})$ with periodic agitation of the mixture with a glass rod. The chromatographic purification of the crude extract was comfortably completed within the four-hour laboratory session by students. Seselin and (+)-epoxysuberosin were isolated in yields of up to $1.1 \% \mathrm{w} / \mathrm{w}$ and $0.9 \% \mathrm{w} / \mathrm{w}$, respectively and isolated samples of both compounds were analyzed by ${ }^{1} \mathrm{H}$ and ${ }^{13} \mathrm{C}$ NMR and FTIR spectroscopy (Figure 2). While students undertook the FTIR spectroscopy experiments and prepared samples for NMR spectroscopy, lab technicians performed NMR spectroscopy experiments. The results obtained by students were consistent with previously published work. ${ }^{11}$

Although this has not been presented in this report, in practice, this experiment also features a second part that challenges students to perform the extraction a plant species that has not been studied employing PHWE (more information is provided in the supporting information).

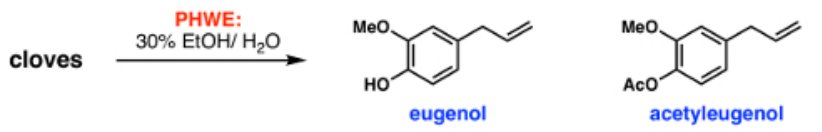

Figure 1. PHWE of cloves. ${ }^{9}$ Please click here to view a larger version of this figure.

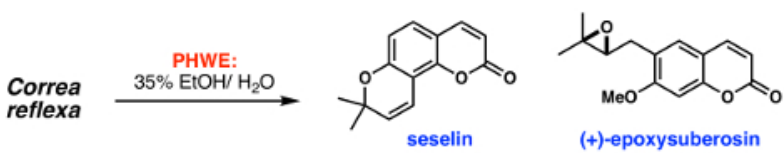

Figure 2. PHWE of Correa reflexa. ${ }^{11}$ Please click here to view a larger version of this figure.

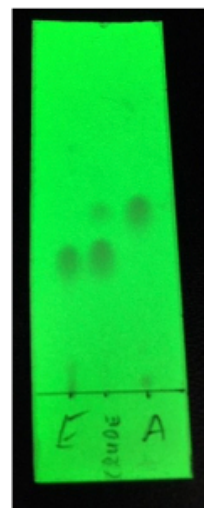

Figure 3. A representative TLC plate prepared by a student. (10\% acetone/ cyclohexane elution). Lane 1 (E): eugenol standard; lane 2 (crude): crude clove extract; lane $3(\mathrm{~A})$ : acetyleugenol standard). Please click here to view a larger version of this figure.

\begin{tabular}{|c|c|c|c|c|c|c|}
\hline $\begin{array}{l}\text { acetonel } \\
\text { cyclohexane (\%v/ } \\
\text { v) }\end{array}$ & $\begin{array}{l}\text { mean acetyl- } \\
\text { eugenol } R_{f}\end{array}$ & $\begin{array}{l}\text { acetyl-eugenol } R_{f} \\
(\sigma)\end{array}$ & mean eugenol $R_{f}$ & eugenol $R_{f}(\sigma)$ & mean $\Delta \mathbf{R}_{f}$ & $\begin{array}{l}\text { number of TLC } \\
\text { analyses }\end{array}$ \\
\hline 0 & 0.06 & 0.08 & 0.04 & 0.06 & 0.02 & 12 \\
\hline 5 & 0.34 & 0.11 & 0.27 & 0.09 & 0.07 & 15 \\
\hline 10 & 0.45 & 0.07 & 0.34 & 0.05 & 0.12 & 20 \\
\hline 20 & 0.51 & 0.07 & 0.41 & 0.06 & 0.10 & 20 \\
\hline 30 & 0.58 & 0.10 & 0.49 & 0.12 & 0.10 & 19 \\
\hline 40 & 0.63 & 0.08 & 0.56 & 0.08 & 0.07 & 16 \\
\hline 50 & 0.76 & 0.08 & 0.73 & 0.08 & 0.03 & 17 \\
\hline 60 & 0.77 & 0.13 & 0.73 & 0.15 & 0.04 & 12 \\
\hline 70 & 0.84 & 0.13 & 0.81 & 0.13 & 0.03 & 11 \\
\hline 80 & 0.90 & 0.06 & 0.87 & 0.08 & 0.02 & 10 \\
\hline 90 & 0.88 & 0.06 & 0.87 & 0.05 & 0.01 & 11 \\
\hline 100 & 0.87 & 0.13 & 0.86 & 0.14 & 0.02 & 6 \\
\hline
\end{tabular}


Table 1. Table of retention factors outlining the effect of eluent composition on $\mathrm{R}_{f}$.

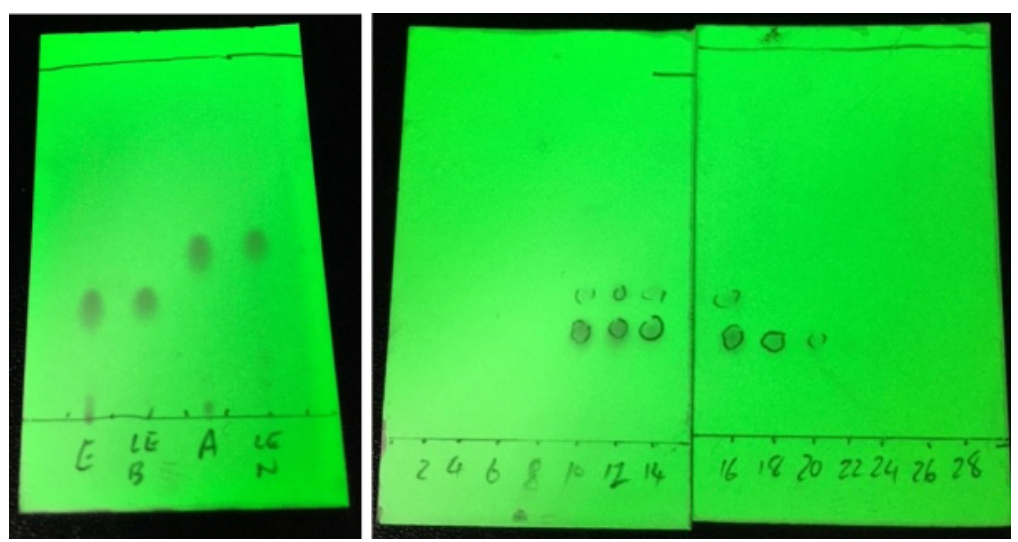

Figure 4. Representative TLC plates prepared by students. Left: A TLC plate analyzing the outcome of the liquid-liquid extraction step (10\% acetone/ cyclohexane elution). Lane $1(E)$ : eugenol reference standard; lane 2 (LEB): eugenol-containing organic extract; lane $3(A)$ : acetyleugenol reference standard; lane 4 (LEN): acetyleugenol-containing organic extract. Right: A TLC plate analyzing the outcome of flash column chromatography step ( $10 \%$ acetone/ cyclohexane elution). Numbers on the TLC plate relate to the test-tube fraction number. Please click here to view a larger version of this figure.

\section{Discussion}

The classical procedure for isolating eugenol from cloves by steam distillation has been part of the intermediate chemistry laboratory program at the University of Sydney for decades but was modernized to employ PHWE methodology in 2016 (Figure 1). ${ }^{9,18}$ This provided a number benefits. Firstly, utilizing household espresso machines in the laboratory environment immediately fascinated and engaged students by illustrating the application of a non-classical, alternative method to effect a traditional scientific study. In addition, this new method reduced the time taken to complete the extraction and enabled the incorporation of additional exercises into this new iteration of the experiment. Specifically, this allowed thin-layer chromatography (TLC) to be introduced (and flash column chromatography for advanced students).

The experiment focusing on the PHWE of cloves was designed as an introductory laboratory experience for second-year undergraduate chemistry students and for this reason it features expository teaching methods. ${ }^{9}$ This more prescriptive, recipe-style procedure allows students with somewhat limited experience in organic chemistry to efficiently complete the extraction of eugenol from cloves. In this experiment, concepts such as acid-base extraction of acidic compounds, utilizing TLC to identify suitable eluent composition for chromatography, and the use of a rotary evaporator are introduced or reinforced by a combination of on-line pre-lab video training and in-person demonstrations. In complementary components undertaken during the two allocated sessions, students in the advanced stream of intermediary chemistry also separated eugenol and acetyleugenol by column chromatography and determined the identity of the extracted components using TLC. In the second session, students could critically compare the two separation methods. In general, students were able to complete the overall experiment within the allocated two four-hour periods with minimal instruction.

The experiment focusing on the PHWE and isolation of seselin and (+)-epoxysuberosin from Correa reflexa was developed for more experienced students third-year undergraduate chemistry students. Notably, this learning exercise was a result of a study originating in the research laboratory. ${ }^{11}$ The first iteration of the experiment was incorporated into the third-year undergraduate chemistry laboratory program at the University of Tasmania in 2015. After two years of revisions and re-evaluation, this experiment was performed by a third-year undergraduate class for the third time in 2017.

This experiment was specifically designed as a guided-inquiry-based activity that strives to simulate some of the approaches employed in natural products research laboratories and features minimal written instructions. This is a student-directed learning experience and the laboratory instructor plays a key role in assisting students as they work through the experiment by providing direction as required. In this experiment, students develop key laboratory skills in chromatography and employ NMR spectroscopy to perform structure elucidation. This laboratory experience reinforces the concept of bioprospecting which is presented to students in the classroom and this can be extended to studies on previously unstudied plant material to provide a more representative experience of natural products bioprospecting. $C$. reflexa is an endemic Australian plant species, however, this sample can be substituted for appropriate leaf material from other terrestrial plant species in this experiment.

\section{Disclosures}

The authors have nothing to disclose.

\section{Acknowledgements}

The authors acknowledge the School of Natural Sciences - Chemistry, University of Tasmania and the School of Chemistry, The University of Sydney for financial support. B.J.D. and J.J. thank the Australian Government for Research Training Program Scholarships. 


\section{References}

1. Newman, D. J., \& Cragg, G. M. Natural Products as Sources of New Drugs from 1981 to 2014. Journal of Natural Products. 79, 629-661 (2016).

2. Barnes, E. C., Kumar, R., \& Davis, R. A. The use of isolated natural products as scaffolds for the generation of chemically diverse screening libraries for drug discovery. Natural Product Reports. 33, 372-381 (2016).

3. DeCorte, B. L. Underexplored Opportunities for Natural Products in Drug Discovery. Journal of Medicinal Chemistry. 59, $9295-9304$ (2016).

4. Bucar, F., Wube, A., \& Schmid, M. Natural product isolation - how to get from biological material to pure compounds. Natural Product Reports. 30, 525-545 (2013).

5. Sticher, O. Natural product isolation. Natural Product Reports. 25, 517-554 (2008).

6. Just, J., Deans, B. J., Olivier, W. J., Paull, B., Bissember, A. C., \& Smith, J. A. New Method for the Rapid Extraction of Natural Products: Efficient Isolation of Shikimic Acid from Star Anise. Organic Letters. 17, 2428-2430 (2015).

7. Caprioli, G., Cortese, M., Cristalli, G., Maggi, F., Odello, L., Ricciutelli, M., Sagratini, G., Sirocchi, V., Tomassoni, G., \& Vittori, S. Optimization of espresso machine parameters through the analysis of coffee odorants by HS-SPME-GC/MS. Food Chemistry. 135, 1127- 1133 (2012).

8. Just, J., Jordan, T. B., Paull, B., Bissember, A. C., \& Smith, J. A. Practical isolation of polygodial from Tasmannia lanceolata.: a viable scaffold for synthesis. Organic Biomolecular Chemistry. 13, 11200- 11207 (2015).

9. Just, J., Bunton, G. L., Deans, B. J., Murray, N. L., Bissember, A. C., \& Smith, J. A. Extraction of Eugenol from Cloves Using an Unmodified Household Espresso Machine: An Alternative to Traditional Steam Distillation. Journal of Chemical Education. 93, 213-216 (2016).

10. Deans, B. J., Bissember, A. C., \& Smith, J. A. Practical Isolation of Asperuloside from Coprosma quadrifida. via Rapid Pressurised Hot Water Extraction Australian Journal of Chemistry. 69, 1219-1222 (2016).

11. Deans, B. J., Just, J., Chetri, J., Burt, L. K., Smith, J. N., Kilah, N. L., de Salas, M., Gueven, N., Bissember, A. C., \& Smith, J. A. Pressurized Hot Water Extraction as a Viable Bioprospecting Tool: Isolation of Coumarin Natural Products from Previously Unexamined Correa (Rutaceae), ChemistrySelect. 2, 2439-2443 (2017).

12. Deans, B. J., Olivier, W. J., Girbino, D., Bissember, A. C., \& Smith, J. A. Extraction of carboxylic acid-containing diterpenoids from Dodonaea viscosa. via pressurised hot water extraction. Fitoterapia. 126, 65-68 (2018).

13. Deans, B. J., Kilah, N. L., Jordan, G. J., Bissember, A. C., \& Smith, J. A. Arbutin Derivatives Isolated from Ancient Proteaceae: Potential Phytochemical Markers Present in Bellendena, Cenarrhenes.and Persoonia.Genera. Journal of Natural Products. 81, 1241-1251, (2018).

14. Deans, B. J., Tedone, L., Bissember, A. C., \& Smith, J. A. Phytochemical profile of the rare, ancient clone Lomatia tasmanica.and comparison to other endemic Tasmanian species L. tinctoria. and L. polymorpha. Phytochemistry. 153, 74-78 (2018).

15. Deans, B. J., Skierka, B., Karagiannakis, B. W., Vuong, D., Lacey, E., Smith, J. A., \& Bissember, A. C., Siliquapyranone: a Tannic Acid Tetrahydropyran-2-one Isolated from the Leaves of Carob (Ceratonia siliqua.) by Pressurised Hot Water Extraction. Australian Journal of Chemistry. 71 (2018).

16. Olivier, W. J., Kilah, N. L., Horne, J., Bissember, A. C., \& Smith, J. A. ent.-Labdane Diterpenoids from Dodonaea viscosa. Journal of Natural Products. 79, 3117-3126 (2016).

17. Rihak, K. J., Bissember, A. C., \& Smith, J. A. Polygodial: A viable natural product scaffold for the rapid synthesis of novel polycyclic pyrrole and pyrrolidine derivatives. Tetrahedron. 74, 1167-1174 (2018).

18. Ntamila, M. S., \& Hassanali, A. Isolation of Oil of Clove and Separation of Eugenol and Acetyl Eugenol. Journal of Chemical Education. 53, 263 (1976).

19. Still, W. C., Kahn, M., \& Mitra, A. Rapid chromatographic technique for preparative separations with moderate resolution Journal of Organic Chemistry. 43, 2923-2925 (1978). 